\title{
In vivo monitoring of function of autologous engineered pulmonary valve
}

\author{
Danielle Gottlieb, MS, MD, MPH, ${ }^{\mathrm{a}, \mathrm{b}}$ Tandon Kunal, BS, ${ }^{\mathrm{a}}$ Sitaram Emani, MD, ${ }^{\mathrm{a}}$ Elena Aikawa, MD, PhD, ${ }^{\mathrm{b}, \mathrm{c}}$

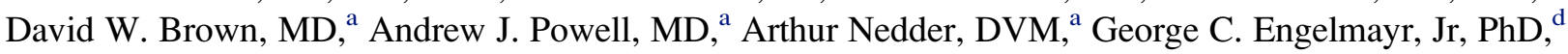 \\ Juan M. Melero-Martin, PhD, ${ }^{\mathrm{a}}$ Michael S. Sacks, PhD, ${ }^{\mathrm{e}}$ and John E. Mayer, Jr, MD ${ }^{\mathrm{a}}$
}

\begin{abstract}
Objectives: Clinical translation of tissue-engineered heart valves requires valve competency and lack of stenosis in the short and long term. Early studies of engineered valves showed promise, although lacked complete definition of valve function. Building on prior experiments, we sought to define the in vivo changes in structure and function of autologous engineered pulmonary valved conduits.
\end{abstract}

\begin{abstract}
Methods: Mesenchymal stem cells were isolated from neonatal sheep bone marrow and seeded onto a bioresorbable scaffold. After 4 weeks of culture, valved conduits were implanted. Valve function, cusp, and conduit dimensions were evaluated at implantation (echocardiography), at the experimental midpoint (magnetic resonance imaging), and at explant, at 1 day, and 1,6,12, or 20 weeks postoperatively (direct measurement, echocardiography). Histologic evaluation was performed.
\end{abstract}

\begin{abstract}
Results: Nineteen animals underwent autologous tissue-engineered valved conduit replacement. At implantation, valved conduit function was excellent; maximum transvalvular pressure gradient by Doppler echocardiography was $17 \mathrm{~mm} \mathrm{Hg}$; most valved conduits showed trivial pulmonary regurgitation. At 6 postoperative weeks, valve cusps appeared less mobile; pulmonary regurgitation was mild to moderate. At 12 weeks or more, valved conduit cusps were increasingly attenuated and regurgitant. Valved conduit diameter remained unchanged over 20 weeks. Dimensional measurements by magnetic resonance imaging correlated with direct measurement at explant.

Conclusions: We demonstrate autologous engineered tissue valved conduits that function well at implantation, with subsequent monitoring of dimensions and function in real time by magnetic resonance imaging. In vivo valves undergo structural and functional remodeling without stenosis, but with worsening pulmonary regurgitation after 6 weeks. Insights into mechanisms of in vivo remodeling are valuable for future iterations of engineered heart valves. (J Thorac Cardiovasc Surg 2010;139:723-31)
\end{abstract}

\begin{abstract}
Although much progress has been made in the treatment of congenital heart disease, valve replacement technologies for children continue to present distinct clinical challenges. For children, currently available bioprosthetic and mechanical valve replacement devices are limited by lack of growth; pediatric patients outgrow valve replacement devices and subsequently require multiple reoperations to accommodate an increasing body size. In addition, whereas valve and patient
\end{abstract}

\footnotetext{
From the Children's Hospital Boston and Harvard Medical School, ${ }^{\text {a }}$ the Massachusetts General Hospital and Harvard Medical School, ${ }^{\mathrm{b}}$ the Brigham and Women's Hospital and Harvard Medical School, ${ }^{\mathrm{c}}$ and the Massachusetts Institute of Technology, ${ }^{\mathrm{d}}$ Boston, Mass, and the University of Pittsburgh, ${ }^{\mathrm{e}}$ Department of Bioengineering, Pittsburgh, Pa.

Disclosures: None.

Funding provided by National Institutes of Health grant RO1 HL089750 (to M.S.S., J.E.M., D.G.), American Heart Association Postdoctoral Fellowship Award (to D.G), and National Institutes of Health grant R01 HL089750 (to J.E.M., D.G.).

Read at the Eighty-ninth Annual Meeting of The American Association for Thoracic Surgery, Boston, Massachusetts, May 9-13, 2009

Received for publication May 10, 2009; revisions received Oct 26, 2009; accepted for publication Nov 2, 2009.

Address for reprints: John E. Mayer, Jr, MD, Bader 273, Children's Hospital, Boston, MA 02115 (E-mail: john.mayer@cardio.chboston.org).

0022-5223/\$36.00

Copyright (c) 2010 by The American Association for Thoracic Surgery

doi:10.1016/j.jtcvs.2009.11.006
}

lifespan are generally well matched in adult populations, the increasing life expectancy of children with congenital heart disease intensifies the requirement for valve durability. Although more durable, mechanical valves are thrombogenic; implantation of a mechanical valve commits a patient to long-term postoperative anticoagulation and its attendant morbidities. In addition, pediatric patients commonly require reconstruction of the pulmonary valve and right ventricular outflow tract, thereby necessitating a valved conduit, a different device from that which is designed for stented or stentless valve replacement applications in adults.

These distinct design inputs for pediatric patients have led to investigation of tissue engineering approaches for pulmonary valve and right ventricular outflow tract replacement. ${ }^{1-3}$ Tissue-engineered heart valves are living tissues offering growth potential and present a possible solution to this challenge. Previous experiments in which autologous cells were seeded onto bioresorbable scaffolds and implanted in an in vivo model have demonstrated the feasibility and potential strengths of this approach. ${ }^{1-3}$

However, insufficient long-term data have been collected to confidently evaluate the long-term function of engineered valves, a requirement for clinical translation. Building on 


\section{Abbreviations and Acronyms \\ ANOVA $=$ analysis of variance \\ DMEM = Dulbecco's modified Eagle's medium \\ FBS = fetal bovine serum \\ MRI = magnetic resonance imaging \\ MSC = mesenchymal stem cell}

prior experiments, we hypothesized that autologous, stem cell-based engineered valved conduits could be successfully implanted into the circulation, with acceptable initial valve function. We sought to understand the process of tissueengineered valved conduit remodeling and to evaluate valve function over time in a large in vivo series. To our knowledge, this series of experiments provides a sufficiently large number of observations to allow the meaningful evaluation of intermediate-term performance of implanted tissueengineered heart valves. We further sought to establish reproducible methods of monitoring in vivo valve function using cardiac magnetic resonance imaging (MRI).

\section{MATERIALS AND METHODS}

Autologous valved conduits were created and implanted into sheep, which were then monitored by cardiac MRI at the experimental midpoint. Animals were grouped and monitored according to the schematic representation shown in Figure 1. Valved conduits were explanted at the experimental end point, within the first 24 hours $(n=7)$, and at $1(n=3), 6(n=3), 12$ $(\mathrm{n}=3)$, or 20 weeks $(\mathrm{n}=3)$ after implantation.

\section{Animals}

Female sheep (Ovis aries, Dorset subspecies) were obtained at 4 weeks of age, weighing approximately $10 \mathrm{~kg}$. Bone marrow aspiration was performed twice on each animal; at 5 and 6 weeks of age, $10 \mathrm{~mL} / \mathrm{kg}$ of bone marrow was harvested from the iliac crest under general anesthesia. Use of experimental sheep was approved by the Institutional Animal Care and Use Committee of Children's Hospital Boston. Animals were cared for by a veterinarian in accordance with the "Guide for the Care and Use of Laboratory Animals."

\section{Cell Isolation and Expansion}

Bone marrow was centrifuged on a Ficoll gradient (Sigma Chemical Co, St Louis, Mo) and the mononuclear fraction was plated on uncoated plates in medium containing Dulbecco's modified Eagle's medium (DMEM) with high glucose, $10 \%$ fetal bovine serum (FBS), 10\% autologous serum, $10 \mathrm{mmol} / \mathrm{L}$ HEPES buffer, and $1 \times$ antibiotic/antimycotic. Mesenchymal stem cells (MSCs) were isolated by their avid adherence to tissue culture plates as previously reported, ${ }^{1,4,5}$ passaged, and expanded in basal medium containing DMEM with high glucose, $10 \%$ FBS, $1 \times$ antibiotic/antimycotic, and 10 $\mathrm{mmol} / \mathrm{L}$ HEPES buffer. Cells were expanded by plating at a density of $10^{4}$ cells $/ \mathrm{cm}^{2}$ and passaged every 3 days when $70 \%$ to $80 \%$ confluence was achieved. Cell passaging continued until approximately 1 billion cells were expanded for each valved conduit. As previously reported, 1 month was required from the harvest of bone marrow to the time of cell seeding on the scaffold. ${ }^{1}$

\section{Scaffold Properties and Assembly}

Sheets of nonwoven scaffold containing $50 \%$ fibers of polyglycolic acid and $50 \%$ fibers of poly-L-lactic acid (Concordia Medical, Warwick, RI) were assembled into a valved conduit by manual and machine needle punch- ing, according to methods established in our laboratory, ${ }^{1}$ based on normal dimensions of the ovine right ventricular outflow tract (data not shown).

\section{Custom Tension Device and Scaffold Preparation for Seeding}

To immobilize the valved conduit, to prevent geometric changes during culture, and to provide a homogeneous surface for cell seeding, we fabricated a custom device for valved conduits in 3-dimensional culture. ${ }^{6}$ The valved conduit scaffold was mounted onto gaskets and tied into position with silk suture material, then sewn circumferentially to the tension device at approximately the level of the cusp base and cusp free edge (Figure 2). The valved conduit was then placed inside a $150-\mathrm{mm}$ glass hybridization bottle and sterilized with ethylene oxide gas. Once sterile, scaffolds were pre-wet with $70 \%$ ethanol, washed 3 times with phosphate-buffered saline, and immersed in a solution of $90 \%$ FBS and $10 \%$ antibiotic/antimycotic (1-2 hours) while cells were prepared for seeding.

\section{Valve Culture}

MSCs were seeded onto the $80-\mathrm{cm}^{2}$ scaffold at a density of 0.5 to $1.7 \times$ $10^{7}$ cells $/ \mathrm{cm}^{2}$ by adding a dense cell suspension to the bottle housing the scaffold. Valves were seeded in $60 \mathrm{~mL}$ of medium containing DMEM high glucose, $20 \%$ FBS, $10 \%$ autologous sheep serum, $2 \times$ antibiotic/antimycotic, 10-mmol/L HEPES buffer, $82-\mu \mathrm{g} / \mathrm{mL}$ ascorbic acid-2-phosphate, and 2-ng/mL basic fibroblast growth factor. During seeding, the scaffold was rotated at $1 \mathrm{rpm}$, and medium was changed every 12 hours. After 72 hours, the valve was removed from the glass bottle and placed in an 850$\mathrm{cm}^{2}$ roller bottle containing $500 \mathrm{~mL}$ of medium as above without sheep serum. The medium was changed every 3 days for 1 month (Figure 2).

\section{Preoperative Blood Preparation for Cardiopulmonary Bypass}

Blood donation bags were prepared with acid-citrate-dextrose buffer for blood banking. An adult sheep was exsanguinated for blood donation; blood was stored at $4{ }^{\circ} \mathrm{C}$ overnight and used for blood prime for cardiopulmonary bypass.

\section{Operation}

Twenty-two autologous valves were prepared according to these methods, 19 of which were implanted in vivo. The remaining 3 valves served as in vitro controls and were analyzed in parallel to characterize tissues at the time of implantation. At operation, a thoracotomy was performed in the fourth intercostal space, and under cardiopulmonary bypass with a beating heart, the native pulmonary valve cusps and a 1- to 2-cm main pulmonary artery segment were excised. The engineered valved conduit was removed from the tension device and interposed between the cut pulmonary artery segments (Figure 2). Epicardial echocardiography was performed to evaluate valve function at implantation (Figure 3). Maximum transvalvular pressure gradient was obtained by continuous-wave spectral Doppler methods. In addition, a semiquantitative evaluation of proximal pulmonary regurgitation was performed, on the basis of vena contracta color jet width and the presence of retrograde diastolic flow in the main pulmonary artery: trivial $=$ long-axis color jet width $\leq 1 \mathrm{~mm}$; mild $=$ color jet width between 1 and $2 \mathrm{~mm}$; moderate = color jet width $>2 \mathrm{~mm}$ but $<50 \%$ of the conduit width; severe $=$ color jet width $>50 \%$ of conduit width and presence of retrograde diastolic flow in the main pulmonary artery. After chest closure, the animal was transferred from the operating table to a recovery sling, allowed to recover until in hemodynamically stable condition, allowed to wake from anesthesia, and extubated. All tubes and drains were removed before return to the cage on the evening of the operation.

\section{MRI}

At each experimental midpoint, animals underwent cardiac MRI (1.5 T Achieva; Philips, Best, The Netherlands) while under general anesthesia. 


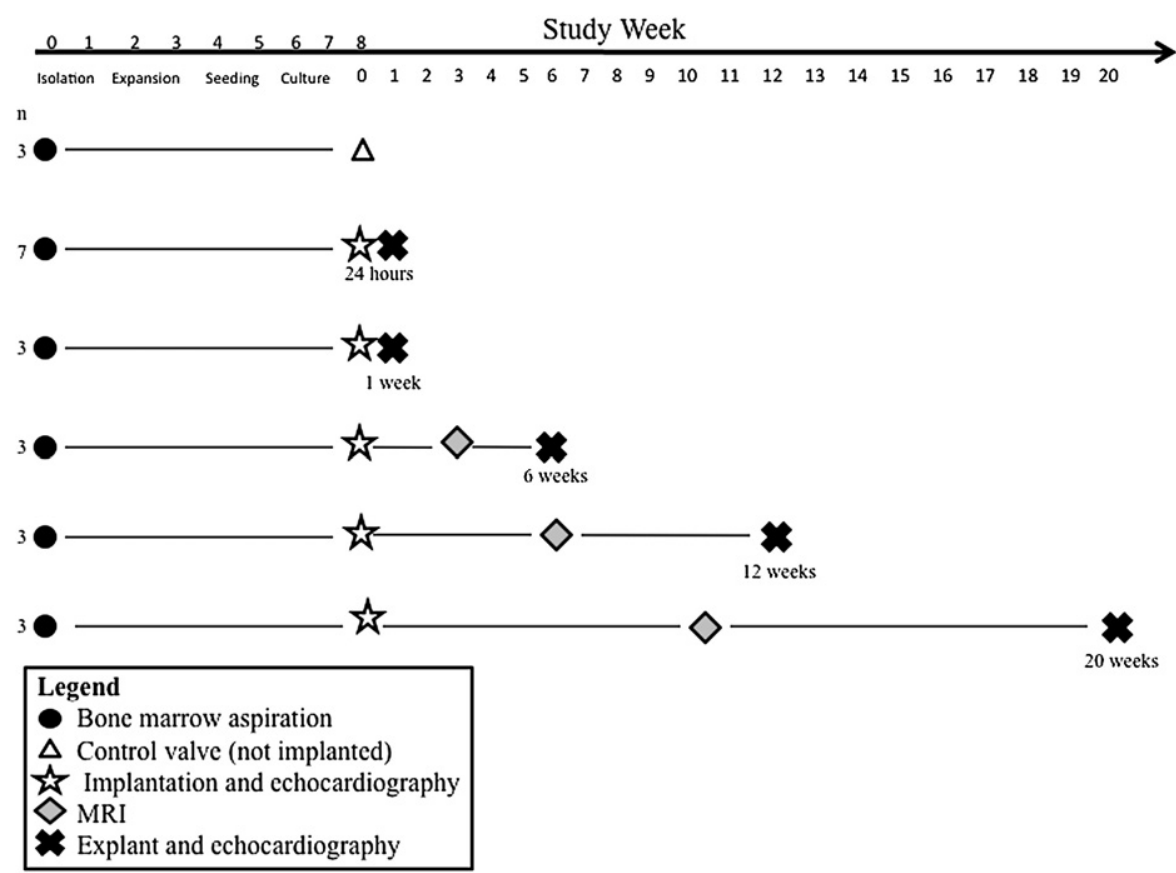

FIGURE 1. Schematic representation of the study design. MRI, Magnetic resonance imaging.

A 5-channel cardiac radiofrequency surface coil was used. After localizing images were obtained, breath-hold electrocardiogram-gated steady-state free precession sequences were obtained in long- and short-axis ventricular planes and in the long- and short-axis planes to the valved conduit. In-plane and through-plane velocities in the valved conduit were measured with an electrocardiogram-gated velocity-encoded cine MRI pulse sequence. Ventricular volumes and conduit flow and velocity data were analyzed offline with commercially available software (Medis QMass and QFlow, Leiden, The Netherlands). The smallest and largest graft diameters were measured to evaluate graft size at each time point.

\section{Valve Explant Procedure}

At each experimental end point, under general anesthesia, epicardial echocardiography was performed for evaluation of valve function, using the same methods as those performed at valve implantation. Pressures were directly measured from positions proximal and distal to the graft to detect pressure gradients. The animal was exsanguinated via an aortic catheter, and the animal's lungs and heart were removed en bloc after cardiac arrest. Smallest conduit diameter and 3 measurements of cusp size were directly measured at the time of explant, including cusp distance from base to free edge (length), free edge distance from commissure to commissure (width), and distance from sinus to cusp free edge (depth). Valves were explanted within 24 hours of implantation $(\mathrm{n}=7)$ and after $1,6,12$, and 20 weeks ( $\mathrm{n}=3$ each).

\section{Histologic Analysis}

Explanted specimens were photographed, dissected, embedded in Tissue-Tek O.C.T. (Sakura Finetek USA, Inc, Torrance, Calif), frozen in 2-methylbutane, and stored until histologic staining. Frozen sections were cut, and slides were stained for overall morphology by hematoxylin and eosin.

\section{Statistical Analysis}

Data were analyzed by the STATA statistical package (STATA IC 10.1, Stata Corporation, College Station, Tex). Analysis of variance (ANOVA) was performed to evaluate statistical significance between groups. Wilcoxon-Mann-Whitney tests were performed to compare means of 2 groups. Mean values are reported as \pm standard deviation. Graphic representations were constructed from data using STATA.

\section{RESULTS}

\section{Valve Fabrication and Implantation}

MSCs were expanded and grown successfully for each animal over $30 \pm 2$ days to reach a total of approximately 1 billion cells (Figure 2,A). During the in vitro culture period, cusp length and width decreased from the time of scaffold assembly to the time of implantation $(3.0 \pm 0.10$ $\mathrm{cm}$ vs $2.5 \pm 0.16 \mathrm{~cm}$ for cusp length, Wilcoxon-MannWhitney $P<.001 ; 2.8 \pm 0.05 \mathrm{~cm}$ vs $1.62 \pm 0.23 \mathrm{~cm}$ for cusp width, Wilcoxon-Mann-Whitney $P<.001$ ). Valved conduit diameter remained stable during the culture period $(2.3 \pm 0.14 \mathrm{~cm}$ before cell seeding vs $2.3 \pm 0.07 \mathrm{~cm}$ at the time of implantation; $P=1.00$ ). Valves were easily removed from the tension device at the time of implantation (Figure 2, $B, C$, and $D$ ). For successful implantation into the pulmonary circulation, all valved conduits contained sufficient extracellular matrix to withstand pulmonary pressure without transmural permeability (Figure 2, $E$ and $F$ ). Conduits retained surgical suture without tearing and exhibited the gross physical appearance of tissue (Figure 2, $E$ and $F$ ).

Animals in the early time point groups ( 24 hours and 1 week) were not humanely killed before explant, but died spontaneously, without evidence of myocardial or valvular dysfunction. Causes of unplanned death included pancytopenia $(\mathrm{n}=2)$, aortic tear $(\mathrm{n}=2)$, carotid artery monitoring line 

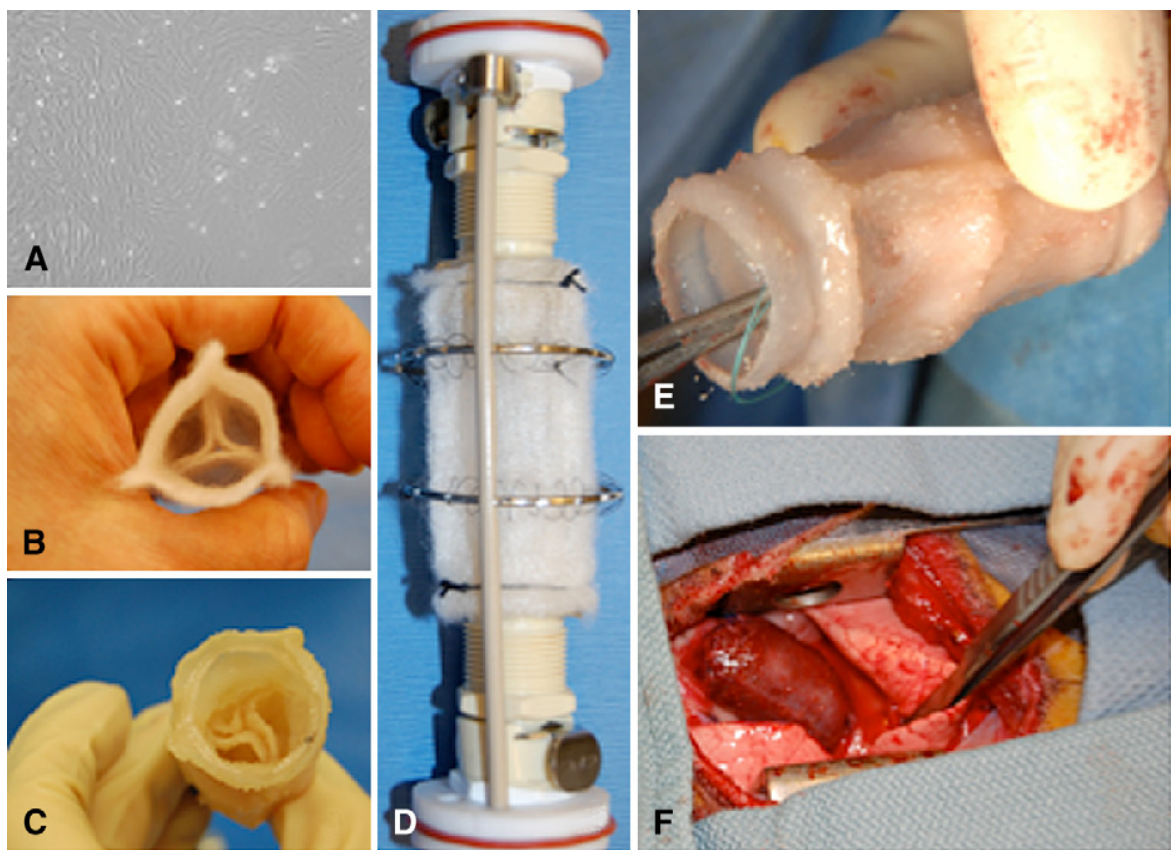

FIGURE 2. A, Phase contrast micrograph $(\times 10)$ of ovine mesenchymal stem cells. B, Assembled valve scaffold before seeding. C, Valved conduit after 1 month of in vitro culture, before implantation. D, Valved conduit mounted and sutured on tension device. E, Valve conduit after removal from tension device and before in vivo implantation. F, Implanted valved conduit.

complication $(\mathrm{n}=1)$, central venous air $(\mathrm{n}=1)$, bleeding $(\mathrm{n}=1)$, and agitation and hypervagal bradycardia during anesthetic emergence $(n=2)$. Pancytopenia was thought to be related to prebypass corticosteroid administration, although not all animals became symptomatic of anemia. The ovine aorta is particularly friable and difficult to cannulate, and subsequent to two aortic tears, the carotid artery was selected for arterial cannulation. Anesthetic emergence difficulties were treated with rectal chloral hydrate $(20 \mathrm{mg} / \mathrm{kg})$, which allowed smooth emergence from anesthesia without agitation or respiratory depression. The remaining animals recovered well without signs of clinical illness. No infectious complications were identified in our study animals.

At implantation, valves demonstrated excellent initial function ( $\mathrm{n}=16$ undergoing echocardiography), with a mean transvalvular Doppler pressure gradient of $17 \pm 4 \mathrm{~mm} \mathrm{Hg}$.



FIGURE 3. Still echocardiographic image in diastole (A) and systole (B) after implantation. Magnetic resonance imaging long view (C) and short view (D) through leaflet cusps at 3 postoperative weeks, showing coapting leaflets in diastole (arrows). Flow image (E) and graphic representation (F) of flow velocities at 3 postoperative weeks showing no regurgitation (x-axis, time; $y$-axis, velocity $[\mathrm{m} / \mathrm{s}]$ ). 



FIGURE 4. A, Measurements of smallest and largest conduit diameters by magnetic resonance imaging. B, Comparison of conduit diameter at time of explant with diameter measured by magnetic resonance imaging. $\mathrm{C}$, Comparison of cusp length (dark circles) and width (open circles) over the study period.

Trivial regurgitation was observed in 11 valves; the remaining 5 demonstrated mild regurgitation at the time of implantation (Figure 3).

\section{Valve Monitoring}

Epicardial echocardiograms performed before death on animals surviving 1 week or less showed valve function, by Doppler pressure gradient and degree of regurgitation, which was equivalent to valve function at the time of implantation. MRI was successfully performed at the study midpoint in 9 animals. By MRI, valvular regurgitation was first detected at 3 weeks. Mean regurgitant fractions and peak velocities measured by MRI ( $\mathrm{n}=3$ at each time point) were as follows: $15.9 \% \pm 9.0 \%$ and $1.6 \pm 0.08 \mathrm{~m} / \mathrm{s}$ at 3 weeks; $32.0 \% \pm 8.0 \%$ and $1.5 \pm 0.4 \mathrm{~m} / \mathrm{s}$ at 6 weeks; and $21.7 \% \pm 8.0 \%$ and $1.1 \pm 0.5 \mathrm{~m} / \mathrm{s}$ at 10 weeks. By MRI, there were no statistical differences between time points $(n=3$ at each time point) with regard to pulmonary regurgitant fraction or transvalvular gradient (ANOVA $P=.13$ for regurgitation and $P=.44$ for gradient). By comparison, aortic valve regurgitant fractions were $3 \%$ or less at all time points. Qualitatively, the cusps moved well and coapted at 3 weeks, but had somewhat restricted motion at 6 weeks, and were fixed and attenuated at 10 weeks. Epicardial echocardiography before planned death in the 6-week animals demonstrated mild to moderate pulmonary regurgitation; at 12 and 20 weeks, animals showed moderate to severe regurgitation.

\section{Valved Conduit Analysis}

Over the in vivo time course of the experiment, the largest and smallest conduit diameters did not change (ANOVA $P=.54$ for smallest and $P=.30$ for largest diameter; Figure 4, $A$ ). When graft diameter measurements were made at similar time points (see Figure 1), there was no difference in the measurements made from MRI images or directly from explanted tissue (ANOVA $P=.52$; Figure $4, B$ ).

At the time of explant of valved conduits in animal groups at 12 weeks or more, dimensional changes of valved conduit cusps were statistically significant, both for length and width measurements (ANOVA at 12 weeks' mean cusp length $1.20 \pm 0.90 \mathrm{~cm}$ vs $2.25 \pm 0.27 \mathrm{~cm}$ at implantation,
$P=.016 ; 12$ weeks' mean cusp width $1.00 \pm 0.30 \mathrm{~cm} \mathrm{vs}$ $1.95 \pm 0.05 \mathrm{~cm}$ at implantation, $P=.001 ; 20$ weeks' mean cusp length $0.67 \pm 0.66 \mathrm{~cm}$ vs $2.25 \pm 0.27 \mathrm{~cm}$ at implantation, $P=.001 ; 20$ weeks' cusp width $0.70 \pm 0.75 \mathrm{~cm} \mathrm{vs}$ $1.95 \pm 0.05 \mathrm{~cm}$ at implantation, $P<0.001)$. Cusp length decreased by $78.5 \%$, and cusp width decreased by $64.1 \%$ from the time of implant to explant at 20 weeks (Figure 4, C).

Implanted and explanted conduits exhibited the gross appearance of tissue (Figure 5). Conduits exhibited a curvature reminiscent of the native pulmonary artery. Three tissue layers, including a center area with residual polymer, were visible at all time points. At 12 weeks in vivo, 3 cusps were visible (Figure 5), although cusp tissue appeared stiff and increasingly attenuated after 12 weeks (Figure 5, $O$ ). The attenuation of cusp tissue over time was evident by the gross appearance of specimens and by hematoxylin-eosin histologic features (Figure 5). Over time, cell and extracellular matrix density increased (Figure 5, $B, E, H, K$, and $N$ ).

\section{DISCUSSION}

In this series of implanted tissue-engineered heart valves, we demonstrate that we could reproducibly fabricate and implant tissue-engineered valved conduits that showed excellent initial valve function and acceptable function at short-term follow-up. MRI provided a noninvasive tool for quantitatively and objectively evaluating valve function. Over time, cusp dimensions decreased and were associated with the development of pulmonary regurgitation. Valved conduit diameter remained stable over 20 weeks in vivo, without evidence of aneurysm formation or stenosis.

Adult progenitor stem cells have been widely investigated in tissue engineering and regenerative medicine as multipotent cell populations having the capacity for self-renewal and differentiation toward multiple differentiated cell lineages. $^{4,5,7-10}$ During fetal life, heart valves develop from delamination of a specialized subpopulation of endothelial progenitor cells that migrate and undergo endothelialto-mesenchymal transdifferentiation and eventually become valve interstitial cells. To date, the steps by which these developmental processes are governed are incompletely 

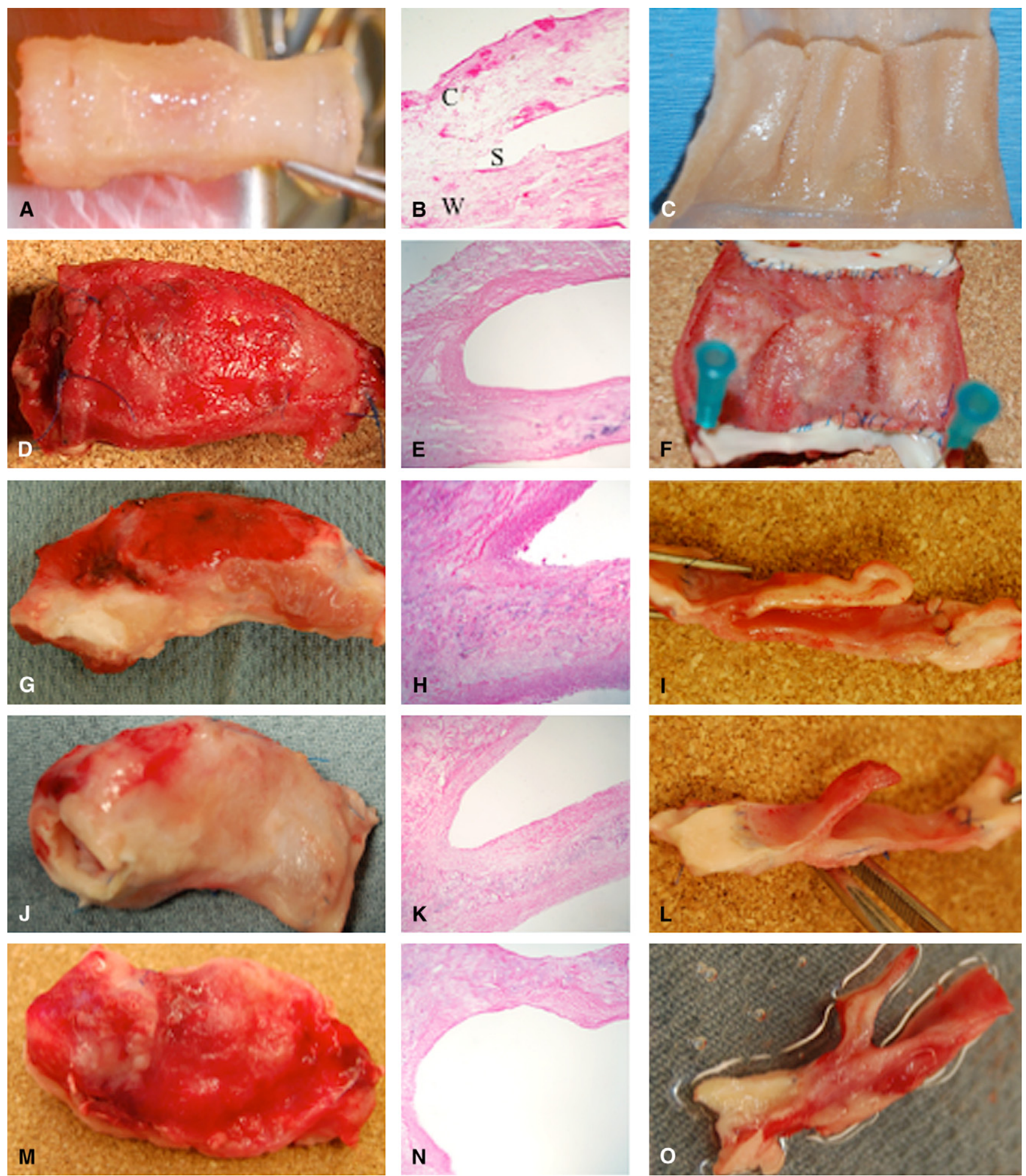

FIGURE 5. Preimplant and explant tissue evaluation showing representative gross and histologic images at time points (A-C) before implant and after 1 (D-F), 6 (G-I), 12 (J-L), and 20 (M-O) weeks in vivo. Histologic images are stained with hematoxylin and eosin, photographed at $\times 20$, and oriented with the cusp at the top and the conduit wall at the bottom of the image ( $C$, cusp; $S$, sinus; $W$, conduit wall).

understood. ${ }^{11,12}$ Owing to the complexity and currently incomplete understanding of heart valve development, tissue engineers have hypothesized that without directly engineering these steps, implanting precursor cells in the appropriate biochemical and mechanical environment may yield similar tissues to those generated in embryologic development. In addition, MSC and other adult progenitor stem cell populations are thought to be immunoprivileged and, as a result, are 
desirable candidates for autologous and potentially allogeneic implants. ${ }^{13}$ MSCs were used in these experiments because they are progenitor cells capable of differentiation into multiple mesenchymal lineages, of which valve interstitial cells represent our target mesenchymal cell population.

We demonstrate a novel device for in vitro culture onto which a valved conduit scaffold can be reproducibly mounted, sterilized, seeded with cells, maintained in 3dimensional culture, and removed for in vivo implantation. In addition, the tension device prevented collapse of the conduit walls during culture and allowed culture of cusps in a closed position.

Valves were harvested at early time points from animals whose deaths were anticipated but not planned. Sheep dying within the first day experienced complications unrelated to the valve, such as aortic cannulation technical errors and difficulties with emergence from anesthesia. Subsequently, technical and anesthetic difficulties were addressed, and at the end of this series, survival was nearly $100 \%$. With sufficient experience with ruminant perioperative care, the sheep model was found to be acceptable for long-term preclinical studies of this tissue-engineered cardiovascular device.

Valved conduit implantation into the circulation requires impermeability to transmural flow across the conduit wall, as well as the ability to hold suture. Valves were cultured as previously described in a proof-of-concept article from our laboratory; valves fabricated in the prior investigation demonstrated these required features. ${ }^{1}$ MSCs served as an autologous source of cell-derived extracellular matrix, providing a barrier to bleeding across the highly porous nonwoven scaffold material. Although sufficient extracellular matrix was formed for hemostasis at the time of implantation, we have yet to understand whether the types of matrix produced by these cells, and the remodeling valved conduits undergo, simulate healthy, normal fetal or postnatal valve matrix turnover. Previous investigations have observed gross similarities in matrix composition between explanted engineered valves and normal valve tissues ${ }^{1-3}$; however, detailed information is not yet available regarding collagen and glycosaminoglycan subtypes. Extracellular matrix molecules and their breakdown products are known to function as regulatory molecules in normal valves and vasculature and are likely to have an influence on the eventual engineered tissue composition. ${ }^{12,14} \mathrm{~A}$ comprehensive and detailed investigation of normal and engineered valve extracellular matrix may yield insight into favorable mechanisms of in vivo engineered valve remodeling.

Heart valves represent a unique application in tissue engineering in that they must demonstrate acceptable hemodynamic function in both the short and long term. To be clinically translatable, reliable methods for monitoring in vivo valve function over time are required. Sheep (Ovis aries) are the most commonly used large animal model in cardiac surgery inasmuch as they have similar hemodynamics to humans and provide rigorous evaluation of long-term valve degradation and calcification. ${ }^{1,3,15}$ Although transthoracic echocardiography has been performed in experimental sheep models by other groups,${ }^{16}$ the anatomy of the ovine thorax provides unreliable echocardiographic windows, which effectively precludes serial echocardiographic monitoring. This limitation of transthoracic echocardiography led to the combined use of MRI and epicardial (open chest) echocardiography.

Here, we demonstrate that cardiac MRI provides a valuable tool for monitoring tissue-engineered heart valve dimensions and function. In patients with tetralogy of Fallot, MRI is currently used for evaluation of valve function over time ${ }^{17}$ We modified our clinical cardiac MRI protocols to capture analogous information from experimental sheep and conclude that these methods provide reliable, quantitative, and noninvasive monitoring of implanted engineered heart valves. Future clinical trials of engineered heart valves should include serial assessment of valve dimensions and function by MRI.

Conduit diameter remained stable over the time course of the study. In contrast to acellular pulmonary conduits previously implanted into the circulation, ${ }^{18}$ these conduits showed no evidence of aneurysm formation. In addition, we saw no evidence of progressive conduit diameter reduction or the development of pressure gradients across the conduit over time. However, we did not observe conduit growth over time, and this observation could represent a true lack of growth or a consequence of a relatively short duration of follow-up. At the time of implantation, the engineered conduit diameter was oversized compared with the native vessel diameter. Twenty weeks after implantation, our longest time point, the engineered vessel diameter more closely matched that of the native vessel. Thus, we were unable to determine whether growth occurred in our engineered conduit.

Histologic characteristics of the valve cusp were similar to previous in vivo results from our laboratory, demonstrating a layered structure in explanted specimens. ${ }^{1}$ We documented increasing valvular regurgitation, corresponding to decreasing cusp length over time. In a recent report, Flanagan and colleagues ${ }^{19}$ report similar leaflet shortening and progressive valve regurgitation of fibrin-based tissue-engineered heart valves in a sheep model. As more is understood about the cellular and molecular mechanisms involved in tissueengineered heart valve remodeling in vivo, the cause of these potentially related observations will be elucidated.

In summary, we build on previous work with a set of reproducible animal experiments, describing longitudinal functional and structural changes in implanted tissue-engineered valved conduits. In addition, we describe effective implant monitoring by cardiac MRI. Reproducible, longitudinal valve function data are required to make progress toward the goal of a clinically implantable tissue-engineered heart valve. Future planned analyses of explanted specimens, 
including detailed histologic, gene, and protein expression profiles of explanted valves, will allow additional insight into cellular and molecular mechanisms of the observed in vivo remodeling of engineered heart valves. Additionally, we plan rigorous evaluation of valves constructed identically, followed by bioreactor conditioning. Although important questions remain regarding the types of scaffold materials and seeded cell types optimally used for heart valve tissue engineering, the knowledge gained from the current experiments will guide the next generation of valve design criteria and future progress toward engineered valves for pediatric application. ${ }^{20}$

We acknowledge the intellectual and technical contributions of Hugo Loyola (statistics and figures), William Regan and the Children's Hospital Boston Perfusion Team (cardiopulmonary bypass), Ryan Conway, Kimberlie Hauser, Mark Kelly, Katie Mullen, and Hugh Simonds (animal care), David Annese (MRI technical support), and Joyce Bischoff (laboratory methods and experimental design), without whom this work would not have been possible.

\section{References}

1. Sutherland FW, Perry TE, Yu Y, Sherwood MC, Rabkin E, Masuda Y, et al. From stem cells to viable autologous semilunar heart valve. Circulation. 2005;111: 2783-91.

2. Hoerstrup SP, Sodian R, Daebritz S, Wang J, Bacha E, Martin DP, et al. Functional living trileaflet heart valves grown in vitro. Circulation. 2000 (19 Suppl 3);III44-9:102.

3. Hoerstrup SP, Cummings I, Lachat M, Schoen FJ, Jenni R, Leschka S, et al. Functional growth in tissue engineered living, vascular grafts: follow-up at 100 weeks in a large animal model. Circulation. 2006;114:II59-66.

4. Perry TE, Kaushal S, Sutherland FW, Guleserian KJ, Bischoff J, Sacks M, et al Thoracic Surgery Directors Association Award. Bone marrow as a cell source for tissue engineering heart valves. Ann Thorac Surg. 2003;75:761-7; discussion 767.

5. Pittenger MF, Mackay AM, Beck SC, Jaiswal RK, Douglas R, Mosca JD, et al. Multilineage potential of adult human mesenchymal stem cells. Science. 1999; 284:143-7.

6. Gottlieb D, Engelmayr GC Jr, Mayer JE Jr. A multi-axial tension apparatus and method for engineering tissues. USSN 61/176,807.

7. Colter DC, Class R, DiGirolamo CM, Prockop DJ. Rapid expansion of recycling stem cells in cultures of plastic-adherent cells from human bone marrow. Proc Natl Acad Sci U S A. 2000;97:3213-8.

8. Silva W, Covas D, Panepucci R, Proto-Siqueira R, Siufi J, Zanette DL, et al. The profile of gene expression of human marrow mesenchymal stem cells. Stem Cells. 2003;21:661-9.

9. McCarty RC, Gronthos S, Zannettino AC, Foster BK, Xian CJ. Characterisation and developmental potential of ovine bone marrow derived mesenchymal stem cells. J Cell Physiol. 2009;219:324-33.

10. Schachinger V, Erbs S, Elsasser A, Haberbosch W, Hambrecht R, Holschermann $\mathrm{H}$, et al. REPAIR-AMI Investigators. Intracoronary bone marrow-derived progenitor cells in acute myocardial infarction. $N$ Engl J Med. 2006;355:1210-21

11. Armstrong EJ, Bischoff J. Heart valve development: endothelial signaling and differentiation. Circ Res. 2004;95:459-70.

12. Lincoln J, Lange AW, Yutzey KE. Hearts and bones: shared regulatory mechanisms in heart valve, cartilage, tendon, and bone development. Dev Biol. 2006; 294:292-302.

13. Uccelli A, Moretta L, Pistoia V. Immunoregulatory function of mesenchymal stem cells. Eur J Immunol. 2006;36:2566-73.

14. Chapados R, Abe K, Ihida-Stansbury K, McKean D, Gates AT, Kern M, et al. ROCK controls matrix synthesis in vascular smooth muscle cells: coupling vasoconstriction to vascular remodeling. Circ Res. 2006;99:837-44.

15. Schoen FJ. Evolving concepts of cardiac valve dynamics: the continuum of development, functional structure, pathobiology and tissue engineering. Circulation. 2008; 118:1864-80.
16. Hopkins RA, Jones AL, Wolfinbarger L, Moore MA, Bert AA, Lofland GK. Decellularization reduces calcification while improving both durability and 1-year functional results of pulmonary homograft valves in juvenile sheep. JThorac Cardiovasc Surg. 2009;137:907-13.

17. Wald R, Redington AN, Pereira A, Provost YL, Paul NS, Oechslin EN, et al. Refining the assessment of pulmonary regurgitation in adults after tetralogy of Fallot repair: should we be measuring regurgitant fraction or regurgitant volume? Eur Heart J. 2009;30:356-61.

18. Leyh RG, Wilhelmi M, Rebe P, Ciboutari S, Haverich A, Mertsching H. Tissue engineering of viable pulmonary arteries for surgical correction of congenital heart defects. Ann Thorac Surg. 2006;81:1466-71.

19. Flanagan TC, Sachweh JS, Frese J, Schnoring H, Gronloh N, Koch S, et al. In vivo remodeling and structural characterization of fibrin-based tissue-engineered heart valves in the adult sheep model. Tissue En Part A. 2009;15:2965-76.

20. Sacks MS, Schoen FJ, Mayer JE. Bioengineering challenges for heart valve tissue engineering. Annu Rev Biomed Eng. 2009;11:289-313.

\section{Discussion}

Dr Sunjay Kaushal (Chicago, Ill). I really enjoyed that talk. I know these are very difficult experiments to perform. Could you provide more details on the histology? For instance, was there actual elastin formation at 20 weeks or how was the collagen organized?

Dr Gottlieb. At this point we have hypotheses but no evidence, and we are involved in detailed investigation of these specific topics, including the presence or absence of elastin at all time points and a description of the evolution of collagen in explanted tissues, including collagen maturity and fiber orientation. Unfortunately, I do not have those data available today.

Dr Kaushal. Was there growth in these valves over time? I know it is a short period of time, but any hints that the valves were growing?

Dr Gottlieb. We still are limited by sample size. Although this is a large number of sheep for a preclinical study, we are still limited by the statistical power of small samples. Therefore, we saw no difference between the time of implant and the time of explant over 20 weeks. However, we started with conduits that were oversized for the native pulmonary artery, and by the time the 20 -week implants occurred, the native artery diameter and the conduit diameter were matched. We did not observe a time point at which there would be wall stress on the tissue-engineered conduit, which might result in growth.

Dr Michael E. Jessen (Dallas, Tex). How much growth was there in the animal? What kind of weight gain did it have over the 20 weeks?

Dr Gottlieb. We implanted at $20 \mathrm{~kg}$ and explanted at 40 to $45 \mathrm{~kg}$.

Dr. Jessen. So they doubled in size over that time interval?

Dr Gottlieb. That's right.

Dr Jessen. Did you make any measurements of what happened to the actual scaffolding material, the polyglycolic acid or the polyL-lactic acid?

Dr Gottlieb. That is an important point, particularly in light of the area of the leaflet decreasing over time. We wonder whether this represents loss of polymer, extracellular matrix, and/or cells, or whether it represents a contraction or shrinkage of the tissue and a stable number of fibers of the bioresorbable scaffolds that are compressed into a smaller area. These are questions that will undergo investigation in the short term.

Dr David Kalfa (Marseille, France). I really congratulate you for this work, and I have 2 questions for you. First, concerning 
the extracellular matrix Dr Kaushal just spoke about, did you evaluate the presence of elastic fibers in the extracellular matrix, at least on a qualitative way, while waiting for detailed investigation to be performed? Second, were you interested in cellular tracking?

Dr Gottlieb. Both are excellent questions. The elastin question I have already spoken to. We do not have the complete data set yet on elastin in all explanted tissues at all time points, so I am reluctant to give information. But our preliminary data did not show much elastin.

In terms of your second question about tracking cells, in our laboratory previously there were experiments with small-scale scaffolds that were engineered into patches in which cells were tracked. We have undertaken some other experiments on a mouse scale where we can track cells using GFP labels. However, in our sheep model, we require that the valved conduits produce sufficient extracellular matrix to be hemostatic when implanted into the circulation. It is established that introduction of labeling vectors can cause changes in expression of a lot of different genes, not just in the addition of a label. After transfecting our bone marrow-derived MSCs with a GFP label, they made insufficient matrix to be hemostatic in the circulation. Because of this limitation, labeled valved conduits were unable to withstand pulmonary pressure. As a result, we have not undertaken those projects because we have not found a way yet to durably and accurately label cells in a large animal analogous model.

Dr John S. Ikonomidis (Charleston, SC). These results are clearly very encouraging because they illustrate the application of some difficult technology, but at the same time they are disappointing because of the observation of failure of these conduits after only 20 weeks. My first question is, have you followed these animals out longer to track the progression of conduit failure?

Second, can you speculate whether cations will be required to prolong conduit durability?

Dr Gottlieb. Those are tough questions. To the first one, our longest duration in our laboratory to date has been 20 weeks, which is 5 months. We see sufficient pulmonary regurgitation at that point to end experiments. We see right ventricular dilation, so evidence of relatively long-term valve regurgitation. We have not monitored them longer than that. We are hopeful that we will have valve leaflets that function better in the future, and we will then undertake those longer-term experiments.

In terms of the disappointment associated with failure of the valves, I think we can only make progress through an iterative process in which we look at the modes of failure in an engineering approach. Our first analysis now is to try to understand what went wrong. Our second move would be to identify the biology resulting in valve failure, then to manipulate it in vitro, and then to try it again in vivo.

Dr Jolanda Kluin (Utrecht, The Netherlands). Could you briefly compare your results with the results of the 2000 paper by Simon Hoerstrup of your institution?

Dr Gottlieb. Dr Hoerstrup's work involved a polymer of polyglycolic acid that was dip coated in poly-4-hydroxybutyrate and seeded with vascular cells. They were completely differentiated cells from the carotid artery smooth muscle cells and endothelial cells. To start, we had completely different models, both in terms of scaffold polymer and in terms of cell. These variables are nontrivial and can account for many differences that we have seen in our preliminary analysis of these valves. His experiments involved 6 animals, 1 at each of 6 different time points. Our current experiment is different because it involves a greater number of animals at multiple time points. His was the proof-of-concept experiment; our current work represents the test of statistical reproducibility. In the current experiments, we have seen evidence of valve regurgitation, which was potentially similar to the central valvular regurgitation, mentioned but not quantitated in the Hoerstrup paper. This builds on that work or the model that was established in that work with different cell types and different scaffolds.

In terms of histology, we will have an opportunity to do a very in-depth analysis and to report those results separately. 